\title{
Forceps Delivery at the Maternity Ward of Donka National Teaching Hospital Conakry (Guinea): Indications and Maternal-Fœtale Prognosis
}

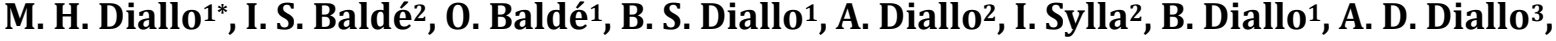 \\ Y. Hyjazi1, N. Keita1 \\ ${ }^{1}$ University Department of Gynecology-Obstetrics, Donka National Hospital, Conakry, Guinea \\ ${ }^{2}$ University Department of Gynecology-Obstetrics, Ignace Deen National Hospital, Conakry, Guinea \\ ${ }^{3}$ University Department of Visceral Surgery, Donka National Hospital, Conakry, Guinea \\ Email: *hadydiallo2002@yahoo.fr
}

How to cite this paper: Diallo, M.H., Baldé, I.S., Baldé, O., Diallo, B.S., Diallo, A., Sylla, I., Diallo, B., Diallo, A.D., Hyjazi, Y. and Keita, N. (2019) Forceps Delivery at the Maternity Ward of Donka National Teaching Hospital Conakry (Guinea): Indications and Maternal-Fœtale Prognosis. Open Journal of Obstetrics and Gynecology, 9, 502-510.

https://doi.org/10.4236/ojog.2019.94049

Received: March 18, 2019

Accepted: April 14, 2019

Published: April 17, 2019

Copyright $\odot 2019$ by author(s) and Scientific Research Publishing Inc. This work is licensed under the Creative Commons Attribution International License (CC BY 4.0).

http://creativecommons.org/licenses/by/4.0/

\section{(C) () Open Access}

\begin{abstract}
Objectives: The objectives of this work were to describe the socio-demographic, clinical and prognostic characteristics of forceps deliveries at the maternity ward of the Donka National Teaching Hospital of Conakry. Parturients and Method: This was a prospective and descriptive study of all forceps deliveries performed at the Donka National Hospital maternity ward over a 6-month period from April 1, 2018 to September 30, 2018. Results: We performed 90 fetal extractions by forceps on 3518 deliveries i.e. a frequency of $2.55 \%$. The socio-demographic profile of the parturient women was that of a young primiparous woman with an average age of 21.20 years and a full-term pregnancy with an average gestational age of 39 weeks of amenorrhoea. Fore position varieties were the most frequent with, in order of frequency, the fore left occipito-iliac (61.3\%) and the fore right occipito-iliac (22.22\%). Posterior varieties accounted for $15.55 \%$ of cases. Clinical pelvimetry showed that $88.89 \%$ of parturients had a practicable pelvis, while $11.11 \%$ had a moderately narrow pelvis. Acute fetal suffering was the most common indication (55.56\%), followed by maternal exhaustion (27.78\%) and prolonged expulsion (16.67\%). All forceps were performed by doctors. We noted 8 cases of forceps failure $(8.88 \%)$ that required a cesarean section. Maternal complications were dominated by soft tissue lesions, including 7 cases of perineal tearing $(7.77 \%) ; 4$ cases of vaginal tearing (4.44\%); 3 cases of cervical tearing (3.33\%) and 2 cases of hemorrhage of delivery by uterine atony (2.22\%). No cases of maternal death have been recorded. The majority of newborns had a normal birth weight $(88.88 \%)$ and more than half of newborns (66.66\%) had an Apgar
\end{abstract}


score below 7 out of 10 at the first minute. By the fifth minute there was an improvement in Apgar's score from an average of 5 to 8 out of 10. Five newborns died, or $5.55 \%$. Conclusion: Forceps extractions are less and less practiced in our maternity wards. Their bad reputation has something to do with it, but this is not always justified, because complications are rare and minimal if the indications are well laid out and the operator experienced. It therefore seems important to reposition this instrument, which still retains its place in obstetrical practices.

\section{Keywords}

Forceps, Delivery, Complications, Conakry

\section{Introduction}

A forceps is a gripping, steering and traction instrument invented by Peter Chamberlain in 1620 and improved by Tarnier in 1877 [1] [2].

A forceps is a metallic instrument in the shape of pliers that allows holding the head of a fetus to facilitate its expulsion. It consists of two branches that are introduced separately and articulated together after setting them up. This instrument may lead to maternal and fetal over-morbidity if the operator is not experienced. As we do not have any recent data on the current situation regarding the use of this instrument in a university hospital in the capital, we decided to carry out this study. According to Cissé et al. in Senegal [3] over the past twenty years, we have observed a progressive decrease in the use of forceps in obstetrical practice in Senegal; this also seems to be the case elsewhere. In the absence of controlled studies in our context, we hypothesize that this finding is certainly related to the expansion of our cesarean section indications and better use of oxytocics.

But one can also blame the inadequacy currently noted in the training of midwives in relation to these instrumental extraction techniques and the bad reputation, whether justified or not, associated with forceps. It is with a view to have a perspective on whether or not to reposition this instrument in our obstetrical practice that we conducted this study to analyze our practice in this area.

The objectives of this situational analysis were: to describe the socio-demographic and clinical characteristics of women involved, to determine the indications and to evaluate the maternal-fetal prognosis.

\section{Parturients and Method}

Our study was conducted in Donka National Hospital, a level III obstetrical reference centre of the maternal health pyramid located in the city of Conakry, which is the first maternity hospital in the country in terms of attendance. This was a prospective and descriptive study of all cases of forceps fetal extraction over a 6-month period, from April 1, 2018 to September 30, 2018. 
We included all women who gave birth by the vaginal route whose expulsion required the application of forceps, a pregnancy term greater than or equal to 37 weeks of amenorrhea (SA), fetus is single, in cephalic presentation and alive at the time of extraction.

The forceps used during this study period was Pajot's forceps.

We have excluded the other instrumental extractions (suction cup).

Maternal variables studied were age, parity, gestational age, the pregnancy follow-up, indications for forceps extractions, the number of failures, maternal complications. In the newborn, we studied the Apgar birth score, the birth weight, neonatal complications such as scalp hematomas, facial skin marks and excoriations, facial paralysis, brachial plexus lesion, anoxic encephalopathy and neonatal death.

In our context, the diagnosis of anoxic encephalopathy is made if the fetus is hypotonic, comatose and after resuscitation, it presents a convulsive seizure.

Perinatal asphyxia is considered to occur when the Apgar score is less than 7 out of 10 at the fifth minute of life. The analysis was manual and our data was entered using Word Excel PowerPoint software, Pack Office 2007, and our results were presented in the form of tables expressed as a percentage and as an average.

Informed consent was obtained from the patients and the investigation was conducted in a confidential and anonymous manner.

Ethics: We obtained the agreement of the national ethics committee

\section{Results}

\subsection{Frequency}

Out of a total of 3518 deliveries, 90 fetal extractions by forceps were performed, representing a frequency of $2.55 \%$.

\subsection{Patient Characteristics}

1) Age: The 16 - 20 age group was the most represented (56.67\%). The average age was 21.20 years with extremes of 16 and 35 years. The distribution by age group is summarized in Table 1.

2) Parity: More than half of the parturient women were primiparous (55.56\%). The average parity was 2 with extremes of 1 and 10. The distribution by parity is summarized in Table 2 .

3) Level of education: The largest number of out-of-school women was parturient (60\%), The distrubution by level of education is summarized in Table 3 .

4) Socio-professional categories: Women in the liberal professions were the most represented (48.89\%), followed by housewives (30\%) and secondary school and university students (21.11\%).

\subsection{Clinical Data}

1) Mode of admission: More than half of the parturient women were evacuated 
Table 1. Distribution of parturient women by age group.

\begin{tabular}{ccc}
\hline Age groups (years) & Number of cases & $\%$ \\
\hline $16-20$ & 51 & 56.67 \\
$21-25$ & 32 & 35.56 \\
$26-30$ & 4 & 4.44 \\
$31-35$ & 3 & 3.33 \\
Total & 90 & 100 \\
\hline
\end{tabular}

Average age $=21.20$ years; Extreme ages $=16$ years and 35 years.

Table 2. Distribution of parturient women by parity.

\begin{tabular}{ccc}
\hline Parity & Number of cases & $\%$ \\
\hline Primiparous (1) & 50 & 55.56 \\
Pauciparous $(2-3)$ & 27 & 30.00 \\
Multiparous $(4-5)$ & 12 & 13.33 \\
Major multiparous (+5) & 1 & 1.11 \\
Total & 90 & 100 \\
\hline
\end{tabular}

Average Parity: 2; Extremes 1 and 10.

Table 3. Distribution of parturients by level of education.

\begin{tabular}{ccc}
\hline Level of Education & Number of cases & $\%$ \\
\hline None & 54 & 60.00 \\
Primary & 8 & 8.88 \\
Secondary & 13 & 14.45 \\
Superior & 15 & 16.67 \\
Total & 90 & 100 \\
\hline
\end{tabular}

(53.34\%), those from their homes represented $33.33 \%$, followed by those who were transferred (13.33\%).

2) Gestational age: The mean gestational age was 39 weeks of amenorrhea with extremes of 37 and 42 weeks of amenorrhea.

3) Prenatal follow-up: Prenatal consultation was performed only once in $83.33 \%$ of cases, twice in $8.89 \%$ of cases, three times in $6.67 \%$ of cases and four times in $1.11 \%$ of cases.

4) Indications of the forceps: The distribution according to the indications is summarized in Table 4.

5) Position Varieties: The varieties of fore positions were mainly found with the following order of frequency: the fore left occipitoiliac (61.3\%) and the fore right iliac occipito (OIDA) (22.22\%). Posterior varieties accounted for $15.55 \%$. Clinical pelvimetry objectified a normal pelvis in $88.89 \%$ of parturients, the pelvis was moderately narrowed in $11.11 \%$ of cases. Fetal suffering was the most frequently reported indication (55.56\%), followed by maternal exhaustion (27.78\%) and prolonged expulsion (16.67\%). 
Table 4. Distribution of parturients according to the forceps indication.

\begin{tabular}{ccc}
\hline Forceps indication & Nombre de cas & $\%$ \\
\hline Acute fetal distress & $\mathbf{5 0}$ & $\mathbf{5 5 . 5 6}$ \\
Extension of the expulsion periode & 15 & 16.67 \\
Stopping fetal progression & 15 & 16.67 \\
Maternal exhaustion & 10 & 11.11 \\
Total & 90 & 100 \\
\hline
\end{tabular}

All forceps applications were performed by physicians.

The extraction by forceps was most often done by a direct hold in occipitopubic $(63.33 \%)$ or occipitosacral $(27.77 \%)$ positions. Oblique positions accounted for $10 \%$ of the cases. An episiotomy was performed on 82 parturients $(91.11 \%)$, the average duration of the procedure in forceps extractions was 17 minutes with extremes of 12 and 30 minutes. We observed 8 cases of forceps failure (8.88\%) that eventually required a cesarean section.

\subsection{Prognosis}

\section{1) Maternal prognosis}

No cases of maternal death were recorded after forceps extractions. Complications were noted in 16 cases (17.17\%). These were

- 7 cases of perineal tearing $(7.77 \%)$;

- 4 cases of vaginal tearings ( $4.44 \%)$;

- 3 cases of cervical tearings (3.33\%);

and 2 cases of hemorrhage of delivery by uterine atony (2.22\%).

\section{2) Fetal prognosis}

The birth weight of newborns averaged 2986 grammes with extremes of 1900 and 4200 . The majority of newborns had a normal weight (88.88\%), macrosomes accounted for $7.77 \%$ and low birth weight $3.33 \%$.

More than half of the newborns (66.66\%) had an Apgar score below 7 out of 10 at the first minute, the average Apgar score at the first minute was 5/10 with extremes of 1 and 9. Hypoxia was found in 28 newborns (31.11\%), 8 of whom were severe (8.88\%). By the fifth minute there was an improvement in Apgar's score, which on average had gone from 5 to 8 out of 10 . Ten newborns (11.11\%) still had moderate hypoxia and 5 died, or $5.55 \%$.

Neonatal complications were represented by scalp haematomas located at the points of support of the spoons with no long-term consequences in 65 newborns (72.22\%), skin marks and excoriations on the face in 9 cases (10\%), subconjunctival hemorrhage in 7 cases or $7.77 \%$, facial paralysis in 1 case $(1.11 \%)$ by compression of the nerve at its emergence from the stylomastoid hole by the posterior spoon spout of the forceps having spontaneously regressed and a lesion of the brachial plexus in 1 case $(1.11 \%)$ in a macrosome having persisted. No lesions were detected in 7 newborns (7.77\%). Neonates were seen again six months after delivery and one [1] had complications related to brachial plexus 
lesions.

\section{Discussion}

\subsection{Frequency}

Since the 1970s, there has been a gradual decrease in instrumental extractions worldwide [3]. In our experience in Conakry, the rate of instrumental forceps extractions is currently $2.55 \%$. This low rate of instrumental extractions is also found in other African series [4] [5] [6].

There are several reasons for this gradual decline in forceps instrumental extractions in our obstetrical practice:

- Birth attendants are less and less trained in these delivery techniques because of their rarity.

- The use of oxytocics is better codified in the treatment of rotation or expulsion defects related to dynamic dystocia.

- The Caesarean section surgery has become a common intervention nowadays, its indications have expanded considerably, especially in the direction of the foetal interest.

\subsection{Socio-Demographic Characteristics}

The socio-demographic profile of women giving birth by forceps in Conakry was that of a young primiparous woman (mean age $=21.20$ years), carrying a full-term pregnancy with a low level of education. This profile is comparable to those reported by studies in Senegal [7], Côte d'Ivoire [5] and Tunisia [6].

\subsection{Clinical Data}

In our study all parturient women were carriers of a full-term pregnancy (100\%) and more than half had a fetus in top presentation in fore left iliac occipito variety (61,3\%), the same observation was made by many authors [4] [5] [7].

The main indications were represented by acute fetal suffering, followed by maternal exhaustion and prolonged expulsion.

The predominance of indications related to poor expulsive efforts and maternal fatigue is reported by several authors [4] [5] [7].

Acute fetal suffering is the first indication in our series with a rate of $55.56 \%$. This rate seems relatively higher compared to the figure reported by Cissé CT in Senegal $21.4 \%$ [3]. It is close to the figures reported by the Collège National des Gynécologues et Obstétriciens Français [8].

Concerning the realization of fetal extraction, forceps is reserved exclusively for physicians [8] [9] [10].

\subsection{Prognosis}

\subsubsection{Maternal Prognosis}

In our series, maternal traumatic complications related to forceps use were dominated by perineal, vaginal and cervical tearings. This finding is found in the 
literature; however our prevalence of tearins is slightly higher than those reported in other studies [11]-[16]. These complications are generally related to a poor appreciation of dilation, with forceps being applied while cervical dilation is incomplete. The risk of vaginal tearing is increased after instrumental extraction compared to spontaneous vaginal extraction by a factor of 3 in the case of forceps [14]. In cohort or comparative studies, reported rates ranged from 2 to $56 \%$ for forceps [13] [14] [16].

These differences are explained by heterogeneous definition criteria and by the many biases found in most retrospective studies.

Vaginal tearings are most often found in the middle and upper thirds. They are favoured by the interposition of vaginal tissue between the instrument and the presentation, by rapid and poorly controlled release or by a significant rotation of the presentation. However, these complications can easily be avoided by carefully controlling the position of the spoons or of the cupula after their insertion and by ensuring that only the presentation is held.

Concerning postpartum hemorrhage, several Cohort studies have shown a significant increase in hemorrhagic risk after instrumental extraction compared to the spontaneous lower route (OR ranging from 1.66 to 2.40 [17] [18] [19] [20] [21]). The reasons given are the increased risk of soft tissue tearing and uterine atony favoured by the presence of associated factors (dystocic labor, prolonged second phase, macrosomy). In the end, everything depends on the strict compliance with the conditions of application, a good selection of indications and a perfect mastery of childbirth techniques.

\subsubsection{Neonatal Prognosis}

In our series more than half (66.66\%) of newborns had an Apgar score below 7 out of 10 in the first minute of life. By the fifth minute there was an improvement in Apgar's score. This observation, which is also found in the literature [5] [7], shows that instrumental forceps extraction does not increase the risk of neonatal suffering if the conditions of application are respected, if the indications are well laid out and if the technique is perfectly controlled. Neonatal morbidity is mainly represented by fetal trauma. The brachial plexus lesion is believed to be due to the macrosomy that caused shoulder dystocia and not to forceps. Other authors have reported the same finding [5] [13] [16]. Other more serious complications are possible with the use of forceps, such as eye damage, paralysis of the brachial plexus and of the facial nerve, and of glitches [3] [10] [12] [13] [14] [15].

The neonatal mortality rate recorded in our series was $5.55 \%$. This rate is high compared to those reported in the literature, which ranges from 0.3 to $0.5 \%$ live births. This difference could be explained by the high frequency of acute fetal suffering in our series.

\section{Limitations and Difficulties}

The scarcity of previous studies is in the study area. 
The results can only be applied to the study site.

Midwives are less and less trained in this delivery technique because of its rarity.

\section{Conclusion}

Instrumental extractions can be very useful in treating expulsion dystocias and/or hastening the birth of a fetus in distress. If the indication is well established, if the conditions of performance are respected and if the operator is trained, the maternal and neonatal prognosis is comparable to that observed in normal vaginal deliveries. It therefore seems useful to reposition these obstetrical techniques in our teachings and in our practice to make parturient women in need benefit from them.

\section{Conflicts of Interest}

The authors declare no conflicts of interest regarding the publication of this paper.

\section{References}

[1] Chang, A.L.S., Scully Noah, M. and Laros, R.K. (2002) Obstetrics Attending Physician Characteristics and Their Impact on Vacum and Forceps Delivery Rates: University of California at San Francisco Experience from 1977 to 1999. American Journal of Obstetrics \& Gynecology, 186, 1299-1303.

https://doi.org/10.1067/mob.2002.123735

[2] Schall, J.P. and Riethmueller, D. (2007) Tears and Incision of the Lower Genital Tract, Instrumental Extraction of the Fetus by Forceps, Suction Cup, Spatulas of Thierry. Mechanics and Obstetrical Techniques. Sauramps Médical, 125-132.

[3] Cissé, C.T., Niang, M.N. and Diouf, A.B. (2015) Indications and Prognosis of Instrumental Fetal Extractions at the IHS Maternity Clinic in Dakar. Journal African de Chirurgie, 3, 186-191.

[4] Ekoundnzola, J.R., Buambo, S., Nkihouabongo, G. and Nayanda, H.F. (2001) Forceps in Africa: About 138 Applications at the University Hospital of Brazaville. Med AFR. Noire, 48, 472-476.

[5] Boni, S., Abauleth, R., Gondo, D., Koffi, A., Effoh, D. and Koné, N. (2005) Indications of Instrumental Extractions and Fetomaternal Prognosis at Cocody University Hospital. J de la Société Africaine de Gynécologie et d Obstétrique, 6, 1-5.

[6] Ben Assia, N., Gara, M.F., Yazidi, M. and Battar, S. (2003) Forceps Childbirth: Indications and Maternal and Fetal Morbidity. Tunisie Médicale, 81, 180-183.

[7] Cissé, C.T., Ewagnignon, E., Fotso, A., Maitournom, F., Moreau, J.C. and Diadhiou, F. (1998) Indications and Prognosis of Forceps Delivery at Dakar Dakar University. Journal of Hospital Medicine, 43, 220-224.

[8] Collège natonal des gynécologues et obstétriciens français (2009) Texte de recommandations. Extractions Instrumentales. La Revue Sage-Femme, 8, 104-107.

[9] Schaal, J.P., Riethmuller, D. and Berthet, J. Instrumental and Manual Maneuvers during Childbirth. Rev Prat, 49, 139-145.

[10] Johnson, J.H., Figueroa, R., Garry, D., Elimian, A. and Maulik, D. (2004) Immediate Maternal and Neonatal Effects of Forceps and Vacuum Assisted Deliveries. Obstetrics \& Gynecology, 103, 513-518. 
https://doi.org/10.1097/01.AOG.0000114985.22844.6d

[11] Allen, V.M., Connell, C.M., Liston, R.M. and Basket, T.F. (2003) Maternal Morbidity Associated with Cesarean Delivery without Labor Compared Spontaneous Onset of Labo at Term. Obstetrics \& Gynecology, 102, 477-482.

[12] Bofill, J.A., Rust, O.A., Schorr, S.J., Brown, A.C., Martin, R.V. and Morrison, J.C.A. (1996) Randomized Prospective Trial of the Obstet Forceps versus the M. Cup Vacuum Extractor. American Journal of Obstetrics \& Gynecology, 175, 1325-1330. https://doi.org/10.1016/S0002-9378(96)70049-2

[13] Caughey, A.B., Sandberg, P.L., Zlanik, M.G., Thiet, M.P., Parer, J.T. and Laros, R.K. (2005) Forceps Compared with Vacuum Rates of Neonatal and Matrernal Morbidity. Obstetrics \& Gynecology, 106, 908-912. https://doi.org/10.1097/01.AOG.0000182616.39503.b2

[14] Gardella, C., Taylor, M., Benedetti, T., Hitti, J. and Critchlow, C. (2001) The Effect of Sequential and Forceps for Assisted Vaginal Delivery on Neonatal and Maternal Out Comes. American Journal of Obstetrics \& Gynecology, 184, 1112-1114.

[15] Kabiru, W.N., Jamieson, D., Raves, W. and Lindsay, M. (2001) Trends in Operative Vaginal Delivery Rates and Associated Maternal Complication Rates in an Inner-City Hospital. American Journal of Obstetrics \& Gynecology, 184, 1112-1114. https://doi.org/10.1067/mob.2001.115178

[16] Lauries, S., Glezerman, M. and Sadan, O. (2005) Maternal and Néonatal Effects of Forceps and Vacuum Operative Delivery. International Journal of Gynecology \& Obstetrics, 89, 293-294.

[17] Combs, C.A., Murphy, E.L. and Lavos, R.K. (1991) Factors Associated with Postpartum Hemorrhage with Vaginal Birth. Obstetrics \& Gynecology, 77, 69-76.

[18] Demissie, K., Rhoads, G.G., Smulian, J.C., Bbalasubramania, B., Joseph, K.S. and Kramer, M. (2004) Operative Vaginal Delivery and Neonatal and Infant Adverse Outcomes: Population Based Retrospective Analysis. BMS, 329, 1-6.

[19] Murphy, D.J., Liebling, R.E., Patel, R., Verity, L. and Swingler, R. (2003) Cohorte Study of Operative's Delivery in the Second Stage of Labour and Standard of Obstetric Care. BJOG, 110, 610-615. https://doi.org/10.1046/j.1471-0528.2003.01463.x

[20] Sheiner, E., Sarid, L., Lery, A., Seidmann, D.S. and Hallak, M. (2005) Obstetric Risk Factors an Outcome of Pregnancies Complicated with Early Postpartum Hemorrhage: A Population Based Study. The Journal of Maternal-Fetal \& Neonatal Medicine, 18, 149-154.

[21] Stones, K.W., Paterson, C.M. and Saunders, N.J. (1993) Risk Factors for Major Obstetric Haemorrhagie. European Journal of Obstetrics \& Gynecology and Reproductive Biology, 48, 15-18. https://doi.org/10.1016/0028-2243(93)90047-G 